\title{
Temporal change in the distribution patterns of hexachlorobenzene and dichlorodiphenyltrichloroethane among various soil organic matter fractions
}

\author{
Jing-jing Zhang ${ }^{a}$, Bei Wen ${ }^{a, *}$, Xiao-quan Shan ${ }^{a, * *}$, Shuzhen Zhang ${ }^{a}$, Shahamat U. Khan ${ }^{b}$ \\ ${ }^{a}$ State Key Laboratory of Environmental Chemistry and Ecotoxicology, Research Center for Eco-Environmental Sciences, \\ Chinese Academy of Sciences, P.O. Box 2871, Beijing 100085, China \\ ${ }^{\mathrm{b}}$ Department of Chemistry and Biochemistry, MSN 3E2, George Mason University, 4400 University Drive, Fairfax, VA 22030-4444, USA
}

Received 12 October 2006; received in revised form 16 January 2007; accepted 20 January 2007

HCB and DDT tend to transfer from FA, HA and BHA fractions to lipid and IR fractions with increasing residence time.

\begin{abstract}
Residence time-dependent distribution patterns of hexachlorobenzene (HCB) and dichlorodiphenyltrichloroethane (DDT) among different soil organic matter fractions of three Chinese soils were investigated. Soil organic matter (SOM) was fractionated into fulvic acid (FA), humic acid (HA), bound-humic acid (BHA), lipid, and insoluble residue (IR) fractions using methyl isobutyl ketone (MIBK) method. Results revealed that as the residence time prolonged, the amounts of HCB and DDT in the FA, HA and BHA fractions decreased, while those in the lipid and IR fractions increased. One- and two-compartment first order, and one- and two-parameter pore-diffusion kinetic models were used to describe the mobility of HCB and DDT from the FA, HA and BHA fractions. The results suggest that excellent agreements were achieved between the experimental data and fits to the two-compartment first order kinetic model $\left(R^{2}>0.97\right)$. The transfer rates of HCB and DDT followed the order FA $>$ HA $>$ BHA.
\end{abstract}

(C) 2007 Elsevier Ltd. All rights reserved.

Keywords: Temporal distribution pattern; Soil organic matter fractions; Hexachlorobenzene; Dichlorodiphenyltrichloroethane

\section{Introduction}

Hexachlorobenzene (HCB) and dichlorodiphenyltrichloroethane (DDT) are of great concern as typical hydrophobic organic compounds (HOCs) due to their long half-life, bioaccumulative nature and chronic adverse effects on humans and animals. Although the use of DDT is banned in developed countries, it still occurs as an impurity and/or intermediate in the manufacturing process of other pesticides and chlorinated compounds (Qiu et al., 2004).

\footnotetext{
* Corresponding author. Tel: +8610 62849329; fax: +861062923563.

** Corresponding author. Tel: +86 10 62923560; fax: +861062923563.

E-mail addresses: bwen@rcees.ac.cn (B. Wen), xiaoquan@rcees.ac.cn (X.-q. Shan).
}

The persistence of HOCs in soil has been attributed to the aging or sequestration mechanism. Despite the extensive consensus on the residence time effect, the relevant mechanisms still remain a matter of debate. The possible mechanisms include intraorganic matter (Cornelissen et al., 2005) and intraparticle diffusion (Steinberg et al., 1987), and transformation of soil organic matter (SOM) (Chen and Xing, 2005). Accordingly, different mathematical models, such as one- and two-compartment first order, two-compartment polymer diffusion and porediffusion kinetics have been proposed (Carroll et al., 1994; Johnson et al., 2001). Understanding of the sequestration processes and the relevant mechanisms that govern HOCs in soil is a prerequisite for prediction of their fate in the environment.

SOM is considered to be the most important factor limiting availability and mobility of HOCs in soil. It has been 
traditionally divided into fulvic acid (FA), humic acid (HA) and humin fractions according to their solubility properties depending on $\mathrm{pH}$. Several investigations have been carried out to elucidate the aging effect on the distribution of HOCs in various SOM fractions. It has been shown that most of HOCs were associated with the humin fraction, while lesser amounts of HOCs were found to be associated with FA and HA fractions (Xie et al., 1997; Kohl and Rice, 1998; Macleod and Semple, 2003). As the contact time increased, the concentration of HOCs in humin fraction increased while those in the FA and HA fractions decreased (Guo and Sun, 2002). Moreover, HOCs associated with humin fraction showed more resistant to desorption and have less bioavailability (Nam and Kim, 2002). The sorption nonlinearity on and desorption hysteresis of HOCs from the humin were more extensively compared with that on HA (Chiou et al., 2000; Kang and Xing, 2005). All these observations suggest that humin plays an important role in HOC sequestration.

Humin is a special form of humic substance tightly associated with mineral matrix, as well as plant and fungal residues in varying stages of decomposition (Rice and MacCarthy, 1989), which may consist of incomplete combustion residues, such as soot, charcoal or char, according to their characteristics. Due to its intractable nature, only very few investigations were carried out to deal with the sorption mechanism of HOCs in humin. Rice and MacCarthy (1989) developed a methylisobutylketone (MIBK) technique for fractionating SOM into FA, HA, BHA, lipid and IR fractions, avoiding degradative losses and chemical changes in one or more of the humin constituents in contrast to digestion with a concentrated $\mathrm{HF} / \mathrm{HCl}$ solution.

The aim of this work was to study the temporal distribution of HCB and DDT among various SOM fractions using the MIBK method in order to better understand the sequestration process of these compounds in soils. To the best of our knowledge this is the first report on the temporal distribution of HOCs among various humin fractions. Four kinetic models were used to describe the transfer of HCB and DDT in various soil organic matter fractions. The implication of the temporal distribution of HCB and DDT to the soil environment has been discussed.

\section{Materials and methods}

\subsection{Soil samples}

Soil samples collected from Heilongjiang, Jiangxi, and Xinjiang provinces of China were air-dried, ground, and passed through a $2 \mathrm{~mm}$ sieve to remove stones, plant roots and other large particles. The soils were sterilized by gamma irradiation $(2.5 \mathrm{Mrad})$ from a ${ }^{60} \mathrm{Co}$ source, and stored under sterilized condition at room temperature for subsequent analysis. Soil organic matter was determined by the Walkley-Black procedure (Nelson and Sommers, 1982). Soil $\mathrm{pH}$ was measured at a soil to $0.01 \mathrm{M} \mathrm{CaCl}_{2}$ solution ratio of $1: 1$ $(w / v)$. Cation-exchange capacity (CEC) was determined by the $\mathrm{BaCl}_{2}$ method (Hendershot and Duquette, 1986).

\subsection{DDT and $H C B$ spiking}

One hundred gram of each air-dried Heilongjiang, Jiangxi, and Xinjiang soil samples with the water contents of 1.9, 2.2 and $1.1 \%$, respectively, in triplicate, were aseptically added to individual flask and then $200 \mu \mathrm{g}$ of HCB and DDT in $10 \mathrm{ml}$ hexane were added to the soils. The soil samples were mixed thoroughly, placed in a hood to evaporate hexane for $5 \mathrm{~h}$, and subsequently sealed in solventrinsed microcosms (1 kg Kilner jar). After sterilization, the microcosms were incubated at $20^{\circ} \mathrm{C}$ for different time intervals. The jars were sealed and stored in the dark to prevent HCB and DDT loss through volatilization and/or photodegradation over the period of incubation. During the course of this study, the soil water contents were determined at time intervals and the relative standard deviations (\% RSD) were 4.9, 3.1 and $10.4 \%$ for Heilongjiang, Jiangxi and Xinjiang soils, respectively.

The accuracy and homogeneity of spiking, the concentrations of HCB and DDT after aging and the mass balance after SOM fractionation were evaluated by analyzing six subsamples as described below.

\subsection{Fractionation of humic substances}

SOM were fractionated into FA, HA, BHA, lipid, and IR fractions by the MIBK technique modified by Kohl and Rice (1998). The whole procedure is summarized in Fig. 1.

Briefly, $10 \mathrm{~g}$ incubated soil sample was first mixed with $200 \mathrm{ml}$ of $0.5 \mathrm{M}$ $\mathrm{NaOH}$ and shaken under nitrogen for $24 \mathrm{~h}$. The sample was centrifuged for 30 min and the supernatant containing FA and HA was separated. The residue precipitate was then briefly stirred with an additional $50 \mathrm{ml}$ of $0.5 \mathrm{M} \mathrm{NaOH}$, allowed to stand for $5 \mathrm{~min}$, and then centrifuged for $30 \mathrm{~min}$. The supernatant containing FA and HA was combined with FA and HA obtained from the previous procedure. This stir and centrifugation operation was repeated five times. FA and HA were further separated by acidifying to $\mathrm{pH}<2$ with concentrated $\mathrm{HCl}$ and centrifuging. The aqueous phase was referred to as FA and the precipitate was HA.

The humin fraction associated with the extracted soil residue was transferred to a separatory funnel along with $200 \mathrm{ml}$ of de-ionized water and $200 \mathrm{ml}$ of MIBK. The aqueous phase was acidified to about $\mathrm{pH} 2$ by concentrated $\mathrm{HCl}$, and then the mixture was shaken vigorously for about $1 \mathrm{~min}$, and allowed to equilibrate for about $1 \mathrm{~h}$. The aqueous phase containing FA and precipitate was separated from MIBK and centrifuged. FA was combined with FA fraction obtained above. The residue precipitate was transferred with $200 \mathrm{ml}$ of $0.5 \mathrm{M} \mathrm{NaOH}$ to the same separatory funnel, shaken vigorously and allowed to stand for $1 \mathrm{~h}$. BHA entered the alkaline aqueous phase, and the aqueous phase and the precipitate were separated from the MIBK phase, and then centrifuged. After that the precipitate was returned to the separatory funnel again with $200 \mathrm{ml}$ of de-ionized water, shaken vigorously for about $1 \mathrm{~min}$, and allowed to equilibrate overnight. After separation the aqueous phase contained BHA, the MIBK phase contained lipid and the precipitate contained IR fraction.

\subsection{Analysis of organic carbon in each SOM fraction}

The organic carbon contents of the FA and BHA fractions were determined by a Phoenix 8000 total organic carbon analyzer (Tekmar-Dohrmann Co.,



Fig. 1. Sequence of the MIBK method. 
Cincinnati, Ohio, USA). While those in the HA, lipid and IR fractions were determined by the Walkley-Black titration (Nelson and Sommers, 1982) after evaporation of all organic solvent and water.

\subsection{Determination of $H C B$ and DDT in soils and humic fractions}

$\mathrm{HCB}\left(\mathrm{C}_{6} \mathrm{Cl}_{6}\right.$, purity $99.9 \%$,) and DDT (2,2-bis (4-chlorophenyl)-1,1,1-trichloroethane, purity $98 \%$, Aldrich Inc., USA) in three soils, and in the HA, BHA and IR fractions were extracted by Soxhlet extraction method. Frozen dried HA, BHA or IR fractions, or $2.00 \mathrm{~g}$ of soil sample was ground with $10.0 \mathrm{~g}$ of anhydrous $\mathrm{Na}_{2} \mathrm{SO}_{4}$ in a mortar and pestle prior to Soxhlet extraction. The ground mixture was placed in a thimble filter and extracted with $100 \mathrm{ml}$ of 1:1 (v/v) hexane/acetone for $24 \mathrm{~h}$ at $5-6 \mathrm{mincycle}^{-1}$. HCB and DDT in FA were extracted with $200 \mathrm{ml}$ of hexane in a separatory funnel for three times with the hexane-water ratio approximately 1:3 (v/v) (Nam and Kim, 2002).

The extracts and MIBK were concentrated to about $1-2 \mathrm{ml}$ by a rotary evaporator, and further purified by column chromatograph using a glass column (12 mm i.d.) loaded with $10 \mathrm{~g}$ activated Florisil (60-100 mesh, Wenzhou Chemical Reagent Factory, China). The elution was subsequently carried out with $10 \mathrm{ml}$ hexane containing $10 \%$ acetone $(\mathrm{v} / \mathrm{v})$, and then concentrated to $1 \mathrm{ml}$ by a rotary evaporator prior to analyses.

For quality assurance, decachlorobiphenyl (DCBP) was added to the fractionated samples before HCB and DDT extraction and purification as a surrogate to monitor the recovery of the operational procedure. The recoveries of the surrogate standards from the FA, HA, BHA, lipid and IR fractions were 89-96, 92-98, 84-91, 97-99, and 91-95\%, respectively, thereby indicating sufficient reliability and validity of the method.

The concentrations of HCB and DDT were analyzed by an Agilent $6890 \mathrm{~N}$ gas chromatograph (Agilent Technologies, Inc., Wilmington, DE, USA) equipped with electron capture detector and a HP-5 fused silica capillary column (30 m length, $0.32 \mathrm{~mm}$ i.d., $0.25 \mu \mathrm{m}$ film thickness, J\&W Scientific Co., Folsom, CA, USA). The oven temperature program started at $60^{\circ} \mathrm{C}$ (holding time $1 \mathrm{~min}$ ), increased to $140^{\circ} \mathrm{C}$ at $15^{\circ} \mathrm{C} / \mathrm{min}$, and finally to $280^{\circ} \mathrm{C}$ at $8{ }^{\circ} \mathrm{C} / \mathrm{min}$ (holding time $5 \mathrm{~min}$ ). Injector and detector temperatures were 220 and $300{ }^{\circ} \mathrm{C}$, respectively. Nitrogen was used as carrier $(2.0 \mathrm{ml} / \mathrm{min})$ and makeup $(60.0 \mathrm{ml} / \mathrm{min})$ gas. A mixed working standard was used for calibration. 2,4,5,6-Tetrachloro- $m$-xylene was used as an internal standard and added before GC-ECD determination. The instrument detection limits of $\mathrm{HCB}$ and DDT were 0.04 and $0.1 \mu \mathrm{g} / \mathrm{l}$, respectively.

\subsection{CPMAS ${ }^{13}$ C NMR spectroscopic study of adsorbents}

The HA, BHA, lipid and IR fractions of Heilongjiang soil were subjected to ${ }^{13} \mathrm{C}$ NMR analysis to obtain their chemical group distribution. All samples were treated with dilute hydrofluoric and hydrochloric acids to reduce ash and paramagnetic contents that would interfere with the acquisition of well-resolved NMR spectra. Solid-state cross-polarization magic-angle-spinning (CPMAS) ${ }^{13} \mathrm{C}$ NMR spectra were acquired on a 300-MHz NMR spectrometer (Varian, San Francisco, USA) as described by Xie et al. (1997).

\subsection{Transfer kinetics models}

Four kinetic models were employed to describe the transfer of HCB and DDT from the FA, HA and BHA fractions. The mathematical expressions are as follows:

\subsubsection{One-parameter spherical pore-diffusion model}

It is assumed that each organic matter fraction is spherical and internally homogeneous porous media. The kinetic of HCB or DDT transfer is described as a radial diffusive penetration of HCB or DDT from these pores:

$\frac{q(t)}{q_{0}}=\frac{6}{\pi^{2}} \sum_{n=1}^{\infty} \frac{1}{n^{2}} \exp \left(\frac{-n^{2} \pi^{2} D_{\mathrm{app}} t}{r^{2}}\right)$

where $q(t)$ is the HCB or DDT concentration in each SOM fraction at a given time $t, q_{0}$ is the initial HCB or DDT concentration in each SOM fraction,
$D_{\text {app }}$ is the apparent diffusion coefficient, $r$ is the sphere equivalent radius, $t$ is the residence time. The model is solved for $\left(D_{\mathrm{app}} / r^{2}\right)$.

\subsubsection{Two-parameter pore-diffusion model}

As well as Eq. (1), the kinetic of HCB or DDT transfer is described as a radial diffusive penetration of HCB or DDT from SOM fraction pores, which is internally heterogeneous. A parameter, $X_{\mathrm{r}}$, served as instantaneous equilibrium (Johnson et al., 2001), is added to Eq. (1):

$\frac{q(t)}{q_{0}}=\frac{\left(1-X_{\mathrm{r}}\right) 6}{\pi^{2}} \sum_{n=1}^{\infty} \frac{1}{n^{2}} \exp \left(\frac{-n^{2} \pi^{2} D_{\mathrm{s}, \mathrm{app}} t}{r^{2}}\right)$

where $X_{\mathrm{r}}$ is the percentage of HCB or DDT of the rapid transfer fraction, $D_{\mathrm{s} \text {,app }}$ is an apparent diffusion coefficient for the slow transfer. The model is solved for $X_{\mathrm{r}}$ and $\left(D_{\text {app }} / r^{2}\right)$.

\subsubsection{One-compartment first order kinetic model}

It is assumed that SOM in each fraction was uniform. HCB or DDT transfer data were fitted by an exponential decay function:

$q(t)=q_{0} \exp (-k t)$

where $k$ is the apparent first order rate constant.

\subsubsection{Two-compartment first order kinetic model}

It is assumed that each organic matter fraction is believed to compose both relatively swollen and condensed humic substances, which are responsible for rapid and slow mass transfer rate, respectively. Simultaneous first order transfer for this system may be expressed mathematically as:

$\frac{q(t)}{q_{0}}=\Phi_{\mathrm{r}} \exp \left(-k_{\mathrm{r}} t\right)+\left(1-\Phi_{\mathrm{r}}\right) \exp \left(-k_{\mathrm{s}} t\right)$

where $\Phi_{\mathrm{r}}$ is the percentage of HCB or DDT of the rapid transfer fraction, while $\left(1-\Phi_{\mathrm{r}}\right)$ is the percentage of the slow transfer fraction, $k_{\mathrm{r}}$ and $k_{\mathrm{s}}$ are the corresponding apparent first order rate constants.

Parameter sets were optimized for all the above noted four equations by nonlinear regression, using the multidimensional simplex method of Nelder and Mead (1965) to minimize a residual sum-of-squares term. Optimization programs were written in $\mathrm{C}++$ using adaptations of code presented by Liu et al. (1995).

\section{Results and discussion}

\subsection{Soil characteristics}

Table 1 summarizes the characteristics of Heilongjiang, Jiangxi, and Xinjiang soils, which differed significantly in the texture and physicochemical properties. The total SOM contents of the three soils varied greatly, with the lowest $(0.97 \%)$ of Xinjiang soil and the highest $(5.48 \%)$ of Heilongjiang soil. Although Jiangxi and Xinjiang soils have similar organic matter contents, their textures are quite different. The clay content of Jiangxi soil is much higher than that of Xinjiang. The fractionation of organic matter of three soils differs significantly. Heilongjiang soil has relatively higher FA $(8.86 \%)$ and HA $(15.36 \%)$ contents. Jiangxi soil has enriched BHA (27.76\%), whereas Xinjiang soil has enriched lipid $(31.4 \%)$ contents. All the soils have IR contents from 40.54 to $47.24 \%$.

The concentrations of HCB, DDT, and DDT degradation products, DDE and DDD in the control soils were lower than the detection limits. 
Table 1

Physicochemical characteristics of Heilongjiang, Jiangxi and Xinjiang soils

\begin{tabular}{|c|c|c|c|}
\hline & \multicolumn{3}{|l|}{ Soils } \\
\hline & Heilongjiang & Jiangxi & Xinjiang \\
\hline Taxonomy & Isohumisols & Ferrisols & Aridisols \\
\hline Organic matter $(\%)^{\mathrm{a}}$ & $5.48(0.10)$ & $1.08(0.06)$ & $0.97(0.03)$ \\
\hline $\mathrm{pH}$ & 7.35 & 4.67 & 7.42 \\
\hline $\mathrm{CEC}(\mathrm{cmol} / \mathrm{kg})^{\mathrm{a}}$ & $25.6(1.0)$ & $14.1(0.4)$ & $10.6(0.8)$ \\
\hline \multicolumn{4}{|c|}{ Particle size distribution } \\
\hline Sand $(\%)$ & 4.4 & 30.9 & 55.2 \\
\hline Silt $(\%)$ & 56.0 & 23.5 & 34.0 \\
\hline Clay $(\%)$ & 39.6 & 45.6 & 10.8 \\
\hline \multicolumn{4}{|c|}{ Organic matter distribution $^{\mathrm{a}}$} \\
\hline $\mathrm{FA}(\%)$ & $8.86(0.44)$ & $5.77(0.21)$ & $6.47(0.29)$ \\
\hline $\mathrm{HA}(\%)$ & $15.36(0.58)$ & $4.71(0.24)$ & $5.69(0.21)$ \\
\hline BHA (\%) & $19.68(0.76)$ & $27.76(0.19)$ & $15.92(0.22)$ \\
\hline Lipid (\%) & $12.32(0.51)$ & $14.52(0.77)$ & $31.41(1.27)$ \\
\hline $\operatorname{IR}(\%)$ & $43.78(2.08)$ & $47.24(2.16)$ & $40.54(1.98)$ \\
\hline
\end{tabular}

a Means of triplicate determinations, with standard deviations in parentheses.

\section{2. ${ }^{13} \mathrm{C}$ NMR spectra of SOM fractions}

The CPMAS ${ }^{13} \mathrm{C}$ NMR spectra and the integration results of the HA, BHA, lipid, and IR fractions of Heilongjiang soil are shown in Fig. 2 and Table 2 as an example. ${ }^{13} \mathrm{C}$ NMR spectra of humic substances are usually divided into chemical shift regions: 0-48 ppm, aliphatic carbon; 48-105 ppm, anomeric carbon; 105-165 ppm, aromatic carbon; and 165-195 ppm, carboxyl carbon. Obviously, basic differences exist among carbon-type distributions in these humic fractions. The lipid fraction had the highest aliphatic carbon, while IR had the highest aromatic carbon. The aliphatic carbon content increased in the order IR $<\mathrm{HA}<\mathrm{BHA}<$ lipid. Compared with HA fraction, BHA had more aliphatic carbon content, and the resonances attributable to anomeric carbon were essentially absent, which is consistent with the previous reports (Xie et al., 1997). The wide range of resonances from 105$195 \mathrm{ppm}$ in the IR fraction demonstrated that both aromatic carbon and carboxyl carbon may be present in this fraction.

\subsection{Spiking of $H C B$ and $D D T$ to soils}

After spiking, the concentrations of HCB and DDT in soils were determined and found to be in the range of 1.90$2.06 \mathrm{mg} / \mathrm{kg}$ for $\mathrm{HCB}$ and $1.82-1.94 \mathrm{mg} / \mathrm{kg}$ for DDT for six replicate determinations, thereby indicating the accuracy and homogeneity of the spiking procedure. At the given residence time intervals of $1,7,14,30,90,120,180$ and $311 \mathrm{~d}$, the contents of HCB and DDT in the spiked soils were found to be $1.74-1.90$ and $1.78-1.88 \mathrm{mg} / \mathrm{kg}$, respectively. These values were slightly but not significantly lower than those of the fresh spiked soil. This indicates that the loss, microbial degradation, as well as the formation of bound residue of HCB and DDT during sequestration could be negligible.

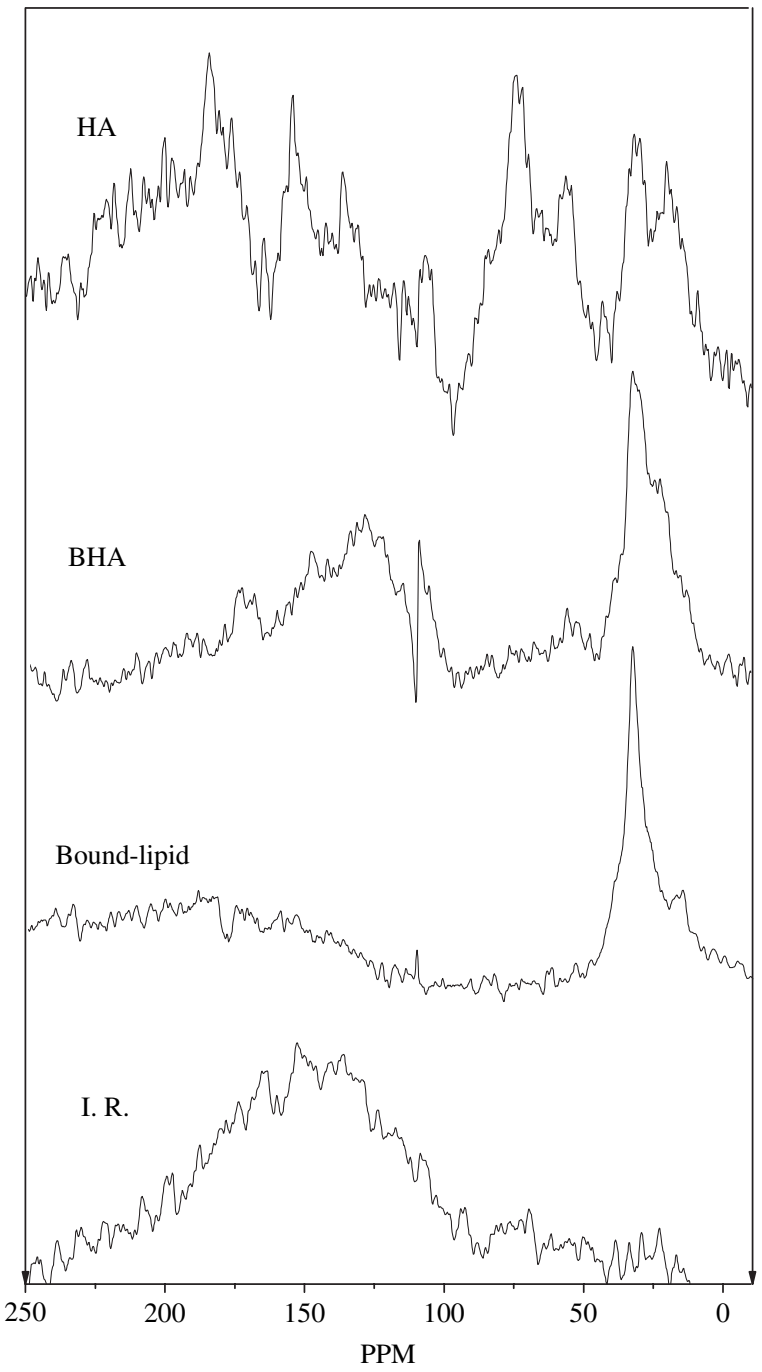

Fig. 2. Solid-state ${ }^{13} \mathrm{C}$ NMR spectra of HA, BHA, lipid, and IR fractions.

\subsection{Recovery of the fractionation and determination of $H C B$ and DDT among various SOM fractions}

In order to evaluate the recovery of the fractionation and determination performance, all soils were subjected to the fractionation and subsequent determination of HCB and DDT immediately after spiking and the solvent evaporated. The amounts of HCB and DDT in six replicated fractionations and their relative standard deviations (\% RSD) are summarized in Table 3. RSD values were in the range of 2.8$18.0 \%$. The sum of the DDT and HCB in the five fractions

Table 2

Integration results of solid-state ${ }^{13} \mathrm{C}$ NMR spectra of the SOM fractions

\begin{tabular}{lllll}
\hline & $\begin{array}{l}\text { Aliphatic C } \\
(0-48 \mathrm{ppm})\end{array}$ & $\begin{array}{l}\text { Anomeric C } \\
(48-105 \mathrm{ppm})\end{array}$ & $\begin{array}{l}\text { Aromatic C } \\
(105-165 \mathrm{ppm})\end{array}$ & $\begin{array}{l}\text { Carboxyl C } \\
(165-195 \mathrm{ppm})\end{array}$ \\
\hline HA & 21.6 & 39.4 & 28.8 & 10.3 \\
BHA & 30.9 & 13.9 & 49.7 & 5.5 \\
Lipid & 76.5 & 1.3 & 14.1 & 8.2 \\
IR & 19.2 & 11.6 & 46.5 & 22.8 \\
\hline
\end{tabular}


Table 3

Reproducibility of fractionation and determination of HCB and DDT in each fraction of unaged soils $(n=6)$

\begin{tabular}{|c|c|c|c|c|c|c|}
\hline & \multicolumn{2}{|c|}{ Heilongjiang soil } & \multicolumn{2}{|l|}{ Jiangxi soil } & \multicolumn{2}{|l|}{ Xinjiang soil } \\
\hline & $\begin{array}{l}\text { Mean } \pm S D \\
(\mu g)\end{array}$ & $\begin{array}{l}\text { RSD } \\
(\%)\end{array}$ & $\begin{array}{l}\text { Mean } \pm S D \\
(\mu g)\end{array}$ & $\begin{array}{l}\text { RSD } \\
(\%)\end{array}$ & $\begin{array}{l}\text { Mean } \pm S D \\
(\mu g)\end{array}$ & $\begin{array}{l}\text { RSD } \\
(\%)\end{array}$ \\
\hline \multicolumn{7}{|l|}{$\mathrm{HCB}$} \\
\hline FA & $0.41 \pm 0.04$ & 10.3 & $0.64 \pm 0.02$ & 3.4 & $0.52 \pm 0.06$ & 11.9 \\
\hline HA & $1.58 \pm 0.13$ & 8.1 & $0.16 \pm 0.03$ & 18.0 & $0.09 \pm 0.004$ & 4.3 \\
\hline BHA & $1.40 \pm 0.11$ & 7.5 & $2.88 \pm 0.29$ & 10.0 & $1.77 \pm 0.05$ & 8.4 \\
\hline Lipid & $11.54 \pm 0.58$ & 5.0 & $9.53 \pm 0.28$ & 2.9 & $13.81 \pm 0.39$ & 2.8 \\
\hline IR & $4.13 \pm 0.29$ & 7.0 & $5.81 \pm 0.27$ & 4.6 & $3.27 \pm 0.40$ & 11.8 \\
\hline Total & $19.06 \pm 0.67$ & 3.5 & $19.02 \pm 0.49$ & 2.6 & $19.46 \pm 0.56$ & 2.9 \\
\hline Sohlet extraction & $19.0 \pm 0.8$ & 4.2 & $20.6 \pm 1.4$ & 6.7 & $20.1 \pm 0.9$ & 4.5 \\
\hline \multicolumn{7}{|l|}{ DDT } \\
\hline FA & $0.44 \pm 0.07$ & 16.2 & $0.35 \pm 0.04$ & 12.1 & $0.43 \pm 0.04$ & 10.2 \\
\hline HA & $1.18 \pm 0.14$ & 12.0 & $0.13 \pm 0.01$ & 8.4 & $0.08 \pm 0.01$ & 13.2 \\
\hline BHA & $1.12 \pm 0.11$ & 9.6 & $2.11 \pm 0.25$ & 12.0 & $0.74 \pm 0.09$ & 12.2 \\
\hline Lipid & $6.16 \pm 0.24$ & 3.9 & $4.88 \pm 0.30$ & 6.1 & $11.23 \pm 0.31$ & 2.7 \\
\hline IR & $9.84 \pm 0.31$ & 3.1 & $11.78 \pm 0.32$ & 2.5 & $6.75 \pm 0.30$ & 4.5 \\
\hline Total & $18.74 \pm 0.44$ & 2.3 & $19.25 \pm 0.51$ & 2.6 & $19.23 \pm 0.44$ & 2.3 \\
\hline Soxhlet extraction & $19.4 \pm 1.1$ & 5.7 & $18.2 \pm 1.2$ & 6.6 & $18.8 \pm 1.4$ & 7.4 \\
\hline
\end{tabular}

is comparable with the total DDT and HCB contents obtained by Soxhlet extraction of the soils, showing good reproducibility of the fractionation and determination performance.

\subsection{Effect of residence time on the distribution of $H C B$ and DDT among various SOM fractions}

The distribution of HCB and DDT among different SOM fractions is shown in Fig. 3. The fractionation time immediately after HCB and DDT spiking and the solvent evaporating was set as zero time. Fast distribution process of HCB and DDT among various humic fractions occurred at zero time. About $10 \%$ of the spiked HCB and DDT were detected in FA and HA fractions for all three soils, while predominant HCB and DDT were found in the lipid and IR fractions. The percentage of $\mathrm{HCB}$ and DDT in the BHA fraction ranged from $10-17$ and 5-13\%, respectively. As the residence time prolonged, redistribution of HCB and DDT among SOM fractions occurred. The contents of HCB and DDT in the FA, HA, and BHA fractions decreased rapidly over the initial $30 \mathrm{~d}$ but decreased more slowly during the following days. Accordingly, HCB and DDT contents in the lipid and IR fractions increased rapidly during the initial $30 \mathrm{~d}$. Then those remained fairly constant in the lipid fraction, while increased slowly until day 120, and then remained almost unchanged in the IR fraction.

The above results revealed a transfer tendency of HCB and DDT from the FA, HA and BHA to the lipid and IR fractions. The same phenomena were also observed by other researchers. Guo and Sun (2002) found that the bound chlorsulfuron residues were decreased in FA and HA, and increased in humin with the increase of incubation time. Bogan and Trbovic (2003) deemed that as the residence time prolonged, the organic contaminants tended to transfer from the outer HA/ FA layer to the "internal surface"-humin, and were then entrapped within humin. Based on NMR measurements and organic contaminant transport, our results suggested that the characteristic of BHA was much more like HA rather than other humin fractions, and may act as an outer layer of soil organic matter.

\subsection{Model fitting of transfer rate parameters}

The one-parameter pore-diffusion model assumes that the soil particles are aggregates of fine mineral grains and natural organic matter. The kinetics of HCB and DDT transfer from each SOM fraction should be described as a radial diffusive penetration from these porous natural particles. The main advantages of this model are that it has only one fitting parameter, $\left(D_{\text {app }} / r^{2}\right)$, and it can theoretically estimate desorption rates a priori based on sorbate and sorbent physical characteristics (Wu and Gschwend, 1986). Only $\left(D_{\mathrm{app}} / r^{2}\right)$ values were calculated in this study because effective diffusion length is often not equal to particle radius. The mass transfer of $\mathrm{HCB}$ and DDT from the FA, HA, and BHA fractions of all three soils was unable to fit one-parameter pore-diffusion model, with the $R^{2}$ in the range of $0.575-0.983$ (FA), $0.483-0.972$ (HA) and $0.323-0.959$ (BHA) (Fig. 4a).

Because the one-parameter pore-diffusion model did not fit entire mass transfer of HCB and DDT, a parameter $X_{\mathrm{r}}$, which represents the instantaneous equilibrium, was added (Johnson et al., 2001). The two-parameter pore-diffusion model, as well as one-parameter pore-diffusion model, only $\left(D_{\text {app }} / r^{2}\right)$ values were calculated. As shown in Fig. 4b, model fits were improved when the parameter $X_{\mathrm{r}}$ was added. However, the twoparameter pore-diffusion model consistently underestimated 

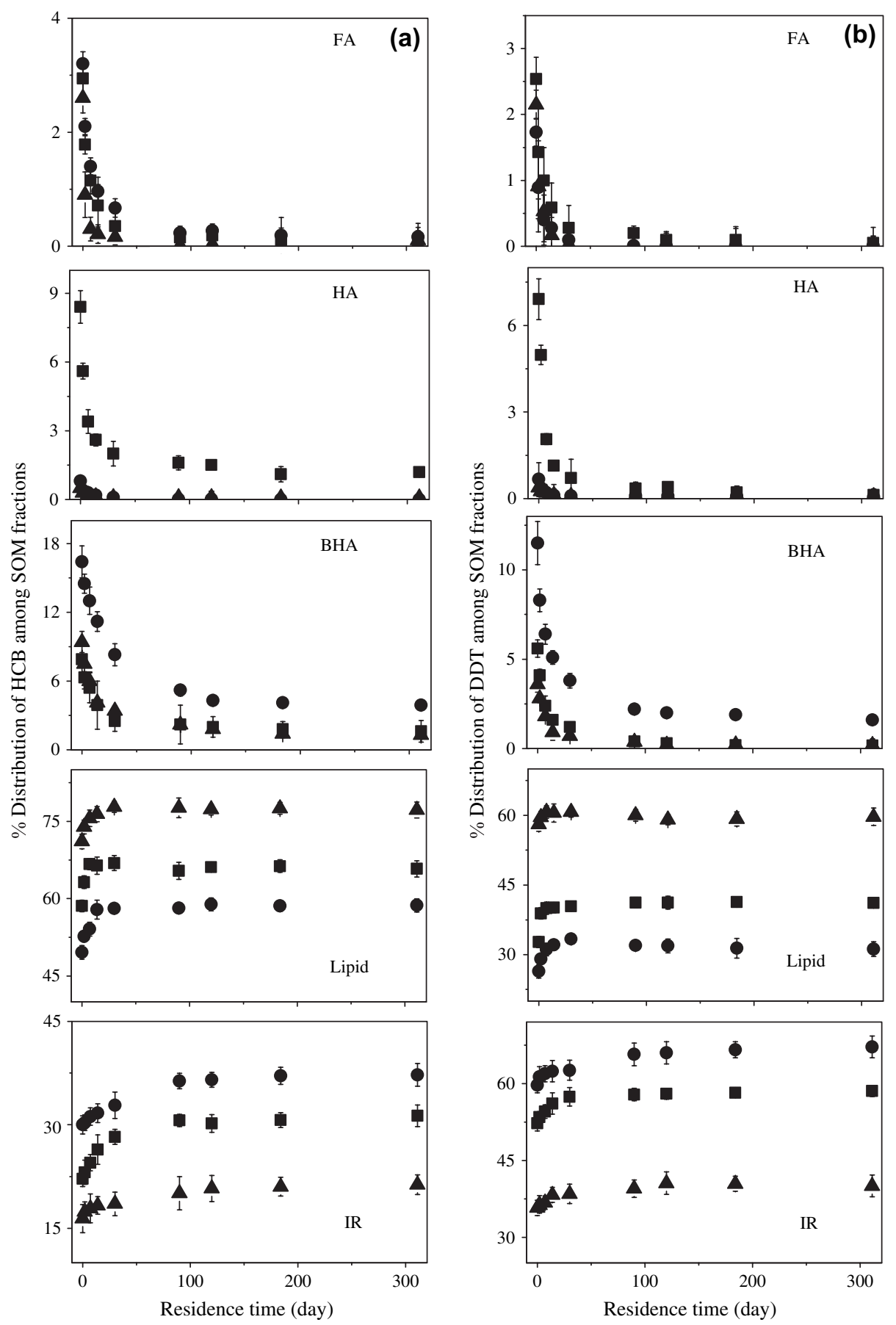

Fig. 3. Distribution patterns of HCB (a) and DDT (b) in each SOM fraction as a function of residence time: ( $\boldsymbol{\square}$ ) Heilongjiang soil, soil. Values are mean \pm standard deviation of six independent experiments of HCB and DDT in a given SOM fraction.

transfer at shorter intermediate times and overestimated transfer at longer intermediate times.

As shown in Fig. 4c, the one-compartment first order kinetic model did not fit the mass transfer of HCB and DDT from the FA, HA, and BHA fractions very well, with the $R^{2}$ in the range of $0.774-0.829$ (FA), $0.344-0.679$ (HA), and 0.324-0.601 (BHA) for HCB, and 0.789-0.949 (FA), $0.459-0.900$ (HA) and $0.255-0.881$ (BHA) for DDT. This indicated the heterogeneity of soil organic matter and different binding sites for HCB or DDT even in the same SOM fraction. Moreover, the decreased correlation coefficients inferred that the heterogeneity followed the order BHA $>\mathrm{HA}>$ FA. The heterogeneous binding sites of FA, progressively extracted humic acids (HAs) and isolated humin for nonpolar compounds were also demonstrated elsewhere (Schlautman and Morgan, 1993; Kang and Xing, 2005). Xu et al. (2006) investigated the structural and adsorption characteristics of HAs isolated from similar soils to ours, red soil, fluvo-aquic soil, black 


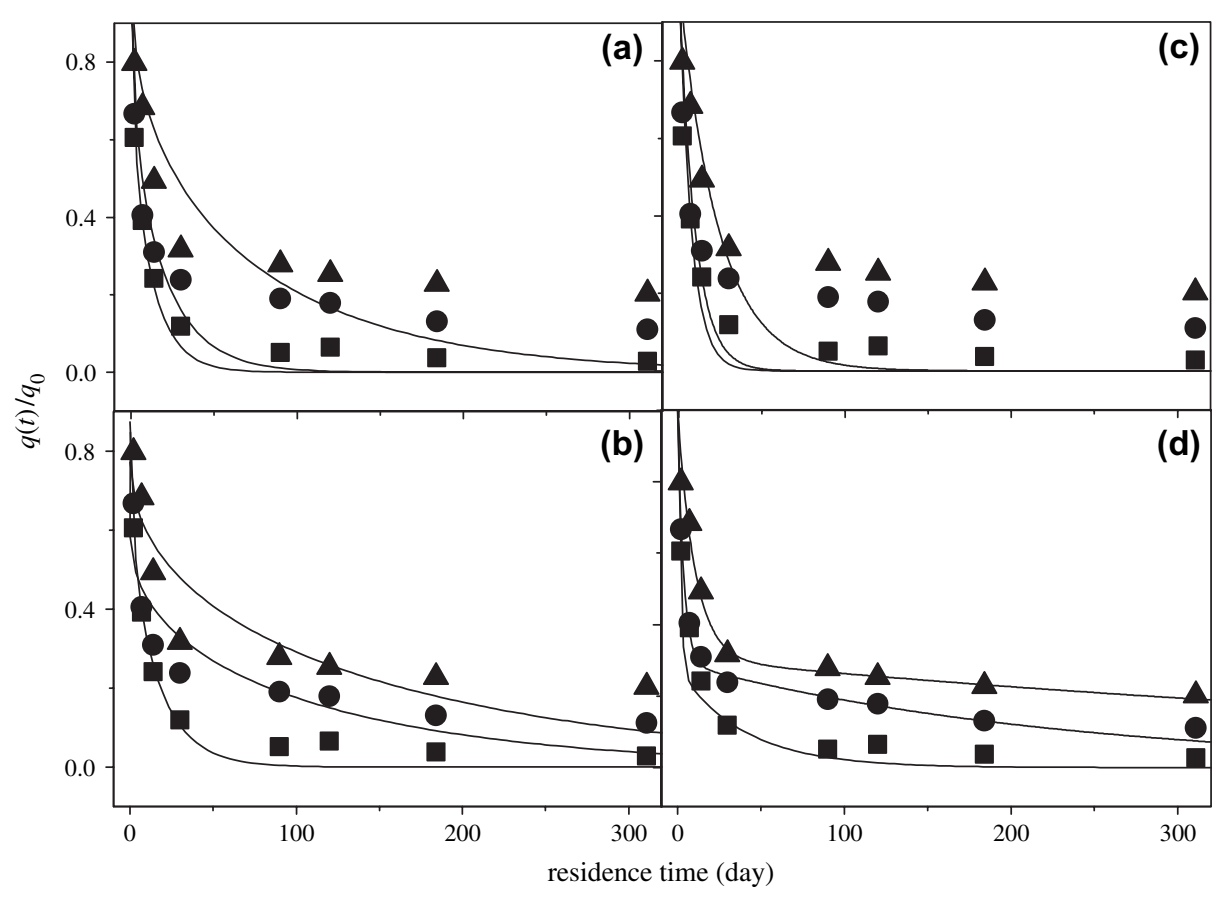

Fig. 4. HCB transfers from FA ( $\boldsymbol{\square})$, HA ( $)$ and BHA ( $\boldsymbol{\Delta}$ ) vs. residence time in Heilongiiang soil. The symbols are experimental data, and the lines are fittings of (a) one-parameter spherical pore-diffusion model, (b) two-parameter pore-diffusion model, (c) one-compartment first order kinetic model, and (d) two-compartment first order kinetic model.

soil and grey desert soil. They found that the HAs from these soils have different contents of chemical groups as aromatic $\mathrm{C}-\mathrm{H}$, aliphatic $\mathrm{C}-\mathrm{H}$, and alicyclic $\mathrm{C}-\mathrm{H}, \mathrm{C}=\mathrm{O}, \mathrm{C}-\mathrm{O}, \mathrm{O}-\mathrm{H}$.

Although the two-compartment first order kinetic model was thought to be largely empirical and inappropriate for characterizing description of sorption kinetics (Carroll et al., 1994), it fits the literature experimental desorption data quite well and is still used to describe the sorption and desorption kinetics (Johnson et al., 2001). This model assumed that mass transfer of HCB or DDT from each SOM fraction was controlled by two simultaneously discrete and independent sites. One is rapid transfer site with $t_{0.5}\left(k_{\mathrm{r}}\right)<10$ days, the time required for $50 \%$ of HCB or DDT associated with a particular reaction transfer from each SOM fraction. The other is much slower transfer site, with $t_{0.5}\left(k_{\mathrm{s}}\right)$ between 11 and 500 days, where $k_{\mathrm{r}}$ and $k_{\mathrm{s}}$ represent the rate constants for the relatively rapid and much slower mass transfer, respectively. In this study, better fits were obtained between the experimental data and the two-compartment first order kinetic model calculation for HCB and DDT transfer from the FA, HA, and BHA fractions of all three soils, with $R^{2}>0.97$ (Table 4 and Fig. 4 d). The $\Phi_{\mathrm{r}}$ and $k_{\mathrm{r}}$ values of FA, HA and BHA, with a few exceptions, followed the order FA $>$ HA $>$ BHA. However, it should be pointed out that it seems unlikely to be the only two types of reaction sites in each SOM fractions. Because of the heterogeneity of each fraction, numerous types of binding sites of HCB or DDT may exist. Apparent mass transfer rate constants may be comprised of numerous diffusional and chemical reaction rates. The optimized rate constants $k_{\mathrm{r}}$ and $k_{\mathrm{s}}$ could only be thought of as representative apparent averages of either relatively rapid or relatively slow reactions. According to the dual-mode sorption model (Pignatello and Xing, 1996; LeBoeuf and Weber, 2000), the sorption to SOM is the sum of partition and numerous adsorption domains, which are analogous to rubbery and glassy polymers. Glassy domains are related to nonlinear adsorption isotherms and sorption-desorption hysteresis due to a hole filling mechanism, whereas rubbery domains are proposed to account for linear sorption. The glass transitions were tested in isolated HA, FA (Hurrass and Schaumann, 2005), lignin (LeBoeuf and Weber, 2000), cellulose (Akim, 1978) and coal (Lucht et al., 1987), which verified that glassiness is a common characteristic of soil organic matter.

However, it is worthwhile considering that HCB and DDT might have been re-distributed during fractionation. Such redistribution may be ascribed to the following two causes: (1) Desorption of HCB and DDT. When $\mathrm{NaOH}$ solution was used to extract FA and HA from humin fractions, HCB and DDT bound to humin fraction might be desorbed and were counted in the FA and HA fractions. In addition, when MIBK was used to fractionate humin, HCB and DDT originally bound to BHA or IR fractions might be extracted by MIBK and counted to lipid fraction. (2) Change in the structure and physicochemical property of SOM. As pointed out by Nam and Kim (2002) that when HA and FA were extracted with $\mathrm{NaOH}$ solution (i.e., $\mathrm{pH}>12$ ), functional groups such as carboxylic groups would predominantly be in a dissociated form. This may produce uncoiled macromolecules of HA and FA in solution and such shift may likely influence the retention of nonpolar hydrophobic contaminants associated with these phases. If this is the case in our study, HCB and DDT initially sorbed to FA and HA fractions might have been re-distributed during the extraction process and re-located to humin fraction. 
Table 4

HCB/DDT transfer rate parameters for two-compartment first order kinetic model

\begin{tabular}{|c|c|c|c|c|c|}
\hline & & $\Phi_{\mathrm{r}}$ & $k_{\mathrm{r}}\left(\right.$ days $\left.^{-1}\right)$ & $k_{\mathrm{s}}\left(\right.$ days $\left.^{-1}\right)$ & $R^{2}$ \\
\hline \multirow[t]{8}{*}{ Heilongjiang soil } & HCB & & & & \\
\hline & FA & $0.724 \pm 0.031$ & $0.644 \pm 0.251$ & $0.050 \pm 0.014$ & 0.971 \\
\hline & HA & $0.706 \pm 0.023$ & $0.284 \pm 0.031$ & $0.004 \pm 0.001$ & 0.987 \\
\hline & BHA & $0.694 \pm 0.047$ & $0.097 \pm 0.016$ & $0.002 \pm 0.001$ & 0.978 \\
\hline & DDT & & & & \\
\hline & FA & $0.811 \pm 0.033$ & $1.226 \pm 0.186$ & $0.058 \pm 0.115$ & 0.986 \\
\hline & HA & $0.781 \pm 0.020$ & $0.273 \pm 0.012$ & $0.015 \pm 0.002$ & 0.997 \\
\hline & BHA & $0.672 \pm 0.027$ & $0.237 \pm 0.021$ & $0.007 \pm 0.002$ & 0.997 \\
\hline \multirow[t]{8}{*}{ Jiangxi soil } & $\mathrm{HCB}$ & & & & \\
\hline & FA & $0.850 \pm 0.018$ & $1.200 \pm 0.142$ & $0.041 \pm 0.006$ & 0.986 \\
\hline & HA & $0.678 \pm 0.050$ & $1.196 \pm 0.406$ & $0.031 \pm 0.005$ & 0.968 \\
\hline & BHA & $0.479 \pm 0.028$ & $0.045 \pm 0.009$ & $0.001 \pm 0.001$ & 0.988 \\
\hline & DDT & & & & \\
\hline & FA & $0.939 \pm 0.013$ & $1.423 \pm 0.112$ & $0.063 \pm 0.006$ & 0.999 \\
\hline & HA & $0.806 \pm 0.022$ & $0.370 \pm 0.041$ & $0.003 \pm 0.001$ & 0.973 \\
\hline & BHA & $0.598 \pm 0.006$ & $0.214 \pm 0.064$ & $0.006 \pm 0.002$ & 0.972 \\
\hline \multirow[t]{8}{*}{ Xinjiang soil } & HCB & & & & \\
\hline & FA & $0.963 \pm 0.005$ & $1.322 \pm 0.061$ & $0.029 \pm 0.008$ & 0.996 \\
\hline & HA & $0.742 \pm 0.036$ & $0.344 \pm 0.066$ & $0.012 \pm 0.001$ & 0.974 \\
\hline & BHA & $0.628 \pm 0.052$ & $0.134 \pm 0.026$ & $0.006 \pm 0.001$ & 0.983 \\
\hline & DDT & & & & \\
\hline & FA & $0.936 \pm 0.011$ & $1.554 \pm 0.172$ & $0.032 \pm 0.011$ & 0.975 \\
\hline & HA & $0.700 \pm 0.042$ & $0.205 \pm 0.012$ & $0.008 \pm 0.003$ & 0.989 \\
\hline & BHA & $0.788 \pm 0.046$ & $0.170 \pm 0.022$ & $0.002 \pm 0.001$ & 0.972 \\
\hline
\end{tabular}

The formation of "bound residues" (unextractable HOCs by exhaustive extraction with nonpolar organic and polar solvents) was well-documented to be increased with the increase of incubation time (Northcott and Jones, 2000). The "bound residues" of DDT in soils are reported to be in the range of $6-25 \%$ of the total added DDT (Xie et al., 1997), and largely affected by the type of soil, environmental conditions, and biological activity (Northcott and Jones, 2000). In this study, the recoveries of $\mathrm{HCB}$ and DDT are relatively high, suggesting that the formation of "bound residue" was insignificant. However, it should be pointed out that the formation of "bound residues" could not be entirely excluded because the bound residue could not be determined directly by our method. About $10 \%$ standard deviations of extraction and determination in this study might shelter the observation of "bound residues".

Nevertheless, the present findings are significant in that they show the relative distribution of aged HCB and DDT among humic substances, especially among humin components. These results demonstrate the highly heterogeneous nature of SOM and the potential role of each humin fraction in the sequestration of HOCs in soil. In the current study, only DDT and HCB, which have similar hydrophobic properties, have been conducted. Future study is needed to test whether our current observation can be generalized for other HOCs with different characteristics.

\section{Conclusions}

Several SOM fractions were experimentally isolated by a MIBK technique. All FA, HA, BHA, lipid and IR fractions showed heterogeneous characteristics of HCB and DDT binding. HCB and DDT were predominantly present in the lipid and IR fractions. Temporal changes in distribution patterns of HCB and DDT among various SOM fractions revealed the movement of HCB and DDT from the FA, HA and BHA to lipid and IR fractions. The good fitness of HCB and DDT transfer from the FA, HA and BHA fractions to two-compartment first order kinetic model indicated heterogeneous binding sites of HCB and DDT in each fraction. The facts that HCB and DDT were associated with water-insoluble lipid and IR fractions implied that HCB and DDT are not readily desorbed to soil water, i.e., HCB and DDT in soil are not labile and not available to plants, soil invertebrates and soil-associated vertebrates and soil microbes.

\section{Acknowledgments}

This study was supported by the National Natural Science Foundation of China (Grants: 20377048 and 20237010).

\section{References}

Akim, E.L., 1978. Cellulose-Bellwether or old hat. Chemtech 8, 676-682. Bogan, B.W., Trbovic, V., 2003. Effect of sequestration on PAH degradability with Fenton's reagent: roles of total organic carbon, humin, and soil porosity. Journal of Hazardous Materials 100, 285-300.

Carroll, K.M., Harkness, M.R., Bracco, A.A., Balcarcel, R.R., 1994. Application of a permeant/polymer diffusional model to the desorption of polychlorinated biphenyls from Hudson River sediments. Environmental Science and Technology 28, 253-258. 
Chen, B., Xing, B., 2005. Sorption and conformational characteristics of reconstituted plant cuticular waxes on montmorillonite. Environmental Science and Technology 39, 8315-8323.

Chiou, C.T., Kile, D.E., Rutherford, D.W., Sheng, G., Boyd, S.A., 2000. Sorption of selected organic compounds from water to a peat soil and its humic acid and humin fractions: potential sources of the sorption nonlinearity. Environmental Science and Technology 34, 1254-1258.

Cornelissen, G., Gustafsson, O., Bucheli, T.D., Jonker, M.T.O., Koelmans, A.A., Noort, P.C.M., 2005. Extensive sorption of organic compounds to black carbon, coal and kerogen in sediments and soils: mechanisms and consequences for distribution, bioaccumulation, and biodegradation. Environmental Science and Technology 39, 6881-6895.

Guo, J., Sun, J., 2002. Studies on bound ${ }^{14} \mathrm{C}$-chlorsulfuron residues in soil. Journal of Agriculture and Food Chemistry 50, 2278-2282.

Hendershot, W.H., Duquette, M.A., 1986. Simple barium chloride method for determining cation exchange capacity and exchangeable cations. Soil Science Society of America Journal 50, 606-608.

Hurrass, J., Schaumann, G.E., 2005. Is glassiness a common characteristic of soil organic matter? Environmental Science and Technology 39, 95349540.

Johnson, M.D., Keinath II, T.M., Weber Jr., W.J., 2001. A distribution reactivity model for sorption by soils and sediments. 14. Characterization and modeling of phenanthrene desorption rates. Environmental Science and Technology 35, 1688-1695.

Kang, S., Xing, B., 2005. Phenanthrene sorption to sequentially extracted soil humic acids and humins. Environmental Science and Technology 39, 134-140.

Kohl, S.D., Rice, J.A., 1998. The binding of contaminants to humin: a mass balance. Chemosphere 36, 251-261.

LeBoeuf, E.J., Weber Jr., W.J., 2000. Macromolecular characteristics of a natural organic matter. 1. Insights from glass transition and enthalpic relaxation behavior. Environmental Science and Technology 34, 3623-3631.

Liu, Y., Kang, J.S., Chen, Y.P., 1995. Nonnumeric Parallel Algorithm-Generic Algorithm. Science Press, Beijing.

Lucht, L.M., Larson, J.M., Peppas, N.A., 1987. Macromolecular structure of coals. 9. Molecular structure and glass transition temperature. Energy and Fuels 1, 56-58.
Macleod, C.J.A., Semple, K.T., 2003. Sequential extraction of low concentrations of pyrene and formation of non-extractable residues in sterile and non-sterile soils. Soil Biology and Biochemistry 35, 1443-1450.

Nam, K., Kim, J.Y., 2002. Role of loosely bound humic substances and humin in the bioavailability of phenanthrene aged in soil. Environmental Pollution 118, 427-433.

Nelder, J.A., Mead, R., 1965. A simplex method for function minimization. Computer Journal 7, 308-313.

Nelson, D.W., Sommers, L.E., 1982. Total carbon, organic carbon, and organic matter. In: Page, A.L., Miller, R.H., Keeney, D.R. (Eds.), Methods of Soil Analysis, Part 2, second ed. ASA and SSSA, Madison, WI, pp. 539-579.

Northcott, G.L., Jones, K.C., 2000. Experimental approaches and analytical techniques for determining organic compound bound residues in soil and sediment. Environmental Pollution 108, 19-43.

Pignatello, J.J., Xing, B., 1996. Mechanisms of slow sorption of organic chemicals to natural particles. Environmental Science and Technology 30, 1-11.

Qiu, X.H., Zhu, T., Li, J., Pan, H.S., Li, Q.L., Miao, G.F., Gong, J.C., 2004 Organochlorine pesticides in the air around the Taihu Lake, China. Environmental Science and Technology 38, 1368-1374.

Rice, J.A., MacCarthy, P., 1989. Isolation of humin by liquid-liquid partitioning. Science of the Total Environment 81/82, 61-69.

Schlautman, M.A., Morgan, J.J., 1993. Effects of aqueous chemistry on the binding of polycyclic aromatic hydrocarbons by dissolved humic materials. Environmental Science and Technology 27, 961-969.

Steinberg, S.M., Pignatello, J.J., Sawhney, B.L., 1987. Persistence of 1,2-dibromoethane in soils: entrapment in intraparticle micropores. Environmental Science and Technology 21, 1201-1208.

Wu, S.C., Gschwend, P.M., 1986. Sorption kinetics of hydrophobic organic compounds to natural sediments and soils. Environmental Science and Technology 20, 717-725.

Xie, H., Guetzloff, T.F., Rice, J.A., 1997. Fractionation of pesticide residues bound to humin. Soil Science 162, 421-429.

Xu, D., Zhu, S., Chen, H., Li, F., 2006. Structural characterization of humic acids isolated from typical soils in China and their adsorption characteristics to phenanthrene. Colloids and Surfaces A: Physicochemical and Engineering Aspects 276, 1-7. 